\title{
The Predictive Value of Preoperative Serum NT- proBNP Levels for the Need for Inotropic Support in the Postoperative Period in Patients Undergoing Coronary Artery Bypass Grafting
}

\author{
Ahmet Öztekin ${ }^{1}$, Mehmet Erdem Memetoðlu², Rasim Kutlu ${ }^{3}$, Ali Ihsan Tekin ${ }^{1}$, Ozan Erbasan1, Ümit \\ Arslan $^{4}$, Ozan Erdem ${ }^{1}$, Özgür Akkaya ${ }^{1}$, Mustafa Şimşek ${ }^{2}$, Murataliev Tolkun Muratalievic ${ }^{5}$ \\ ${ }^{1}$ Akdeniz University School of Medicine, Department of Cardiovascular Surgery, Antalya,Turkey, \\ ${ }^{2}$ Dr. Siyami Ersek Cardiovascular and Thoracic Surgery Hospital, Department of Cardiovascular \\ Surgery, Istanbul,Turkey, ${ }^{3}$ Giresun Universty, Department of Cardiology, Giresun, Turkey, ${ }^{4}$ Atatürk \\ Training and Education Hospital, Department of Cardiovascular Surgery, Erzurum, Turkey, \\ ${ }^{5}$ National Cardiology and Internal Medicine Institude, Department of Cardiology, Bishkek, Kyrgyzstan
}

Keywords:

NT-proBNP, atrial fibrillation, coronary artery disease, inotropic agents.

\begin{abstract}
:
Background: High preoperative BNP levels can be a marker of higher risk for coronary artery bypass grafting. We investigated the predictive value of serum NT-proBNP levels undergoing coronary artery bypass grafting for the need for inotropic support.

Methods: In this prospective study, preoperative serum NT-proBNP levels were obtained in 51 patients [80.4\% ( $n=42)$ were males and $19.6 \%(n=9)$ were females] undergoing isolated coronary artery bypass grafting. The study patients were divided into three groups depending on NT-proBNP levels as low NT-proBNP $(<100 \mathrm{pg} / \mathrm{ml}$, Group-1, 29.4\%, $n=15)$ group, moderately high NT-proBNP $(N T$-proBNP $<500 \mathrm{pg} / \mathrm{mL}$ and $>100 \mathrm{pg} / \mathrm{mL}$, Group-2, 29.4\%, $n=15)$ group, and high NT-proBNP (>500 pg/mL, Group-3, 41.2\%, $n=21)$ group.

Results: At postoperative day 0 , the mean adrenalin, dopamine, dobutamine, and noradrenalin consumptions were $0.1 \pm 0.7 \mathrm{microgram} / \mathrm{kg} / \mathrm{min}, 0.08 \pm 0.56 \mathrm{microgram} / \mathrm{kg} / \mathrm{min}, 2.1 \pm 3.01 \mathrm{microgram} /$ kilogram $/ \mathrm{min}$, and $1.35 \pm 3.45 \mathrm{microgram} / \mathrm{kg} / \mathrm{min}$, respectively. There were no statistically significant differences between the groups in terms of the use of adrenalin $(p=0.50, p>0.05)$, dopamine $(p=0.31$ $p>0.05)$, dobutamine $(p=0.59 p>0.05)$, and noradrenalin $(p=0.24 p>0.05)$ at postoperative day 0 . The doses of inotropic agents used at postoperative days 1 and 2 did not show significant differences between the groups for the three inotropic agents.
\end{abstract}

Conclusion: Preoperative serum NT-proBNP levels in patients undergoing coronary artery bypass grafting are not associated with the need for inotropic support in the postoperative period.

(Cardiovasc.j. 2017; 9(2): 90-96)

\section{Introduction:}

Brain natriuretic peptide (BNP) is a 32 -amino acid polypeptide containing 17 -amino acid residue ring characteristic for all natriuretic peptides. ${ }^{1}$ Cardiac ventricles are the main source of BNP in contrast to ANP for which atriums serve as the main site of storage. For this reason, this polypeptide, unlike other natriuretic peptides, serve as a more sensitive and specific biomarker of ventricular disorders.

Biochemical indicators such as creatine kinase, creatine kinase MB fraction, lactate dehydrogenase, and troponins are used to monitor ischemic injury in coronary artery disease. The common characteristic of these indicators is that all are released by injured cells. Unlike from these, BNP is not only released from dead myocytes, but also from living myocytes as a result of an increase in the ventricular surface tension. To this respect, $\mathrm{BNP}$ is considered to be a more accurate marker reflecting the severity of ischemia in coronary artery disease.

High preoperative BNP levels can be a marker of higher risk for coronary artery bypass grafting.

Address of Correspondence: Ahmet Öztekin, Akdeniz University School of Medicine, Department of Cardiovascular Surgery, Antalya,Turkey. E-mail: dr.rasim@mail.ru 
Sarýbülbül et al. reported a significant relationship between preoperative BNP levels and crossclamping time and need for postoperative inotropic support. ${ }^{2}$ In their study, patients with higher BNP values in the preoperative period were reported to have longer cross-clamping times. Both high BNP levels and prolonged cross-clamping time are associated with the extensiveness of coronary artery disease.

The aim of the present study is to elucidate the relationship between preoperative serum BNP levels and the use of inotropic support in the early postoperative period in patients who underwent coronary artery bypass graft surgery.

\section{Methods:}

The study included a total of 60 patients, who underwent cardiopulmonary bypass graft surgery at our hospital between October 2013 and May 2014. The ethical implications regarding the study were approved by the local Ethics Committee. Consent was obtained from the subjects for participation in the study in line with World Medical Association Declaration of Helsinki (World Medicine Assembly, 2004 Tokyo, Japan). Nine out of 60 patients evaluated for the study did not comply with the inclusion criteria and they were excluded from the study; the data of 51 patients were used in the statistical analysis.

The main preoperative characteristics of patients and intraoperative data are shown in table I. The study patients were divided into three groups depending on NT-proBNP levels as low NTproBNP (<100 pg/mL, Group-1, 29.4\%, n=15) group, moderately high NT-proBNP (NT-proBNP <500 pg/ $\mathrm{mL}$ and $>100 \mathrm{pg} / \mathrm{mL}$, Group-2, 29.4\%, n=15) group, and high NT-proBNP (>500 pg/mL, Group-3, 41.2\%, $\mathrm{n}=21$ ) group. The mean NT-proBNP level was $920.69 \pm 1497.11 \mathrm{pg} / \mathrm{mL}$ in the whole study group, $42.41 \pm 15.25 \mathrm{pg} / \mathrm{mL}$ (maximum value: $68 \mathrm{pg} / \mathrm{mL}$, minimum value: $20.69 \mathrm{pg} / \mathrm{mL}$ ) in Group 1, 221.87士 $131.96 \mathrm{pg} / \mathrm{mL}$ (maximum value: $494 \mathrm{pg} / \mathrm{mL}$, minimum value: $100 \mathrm{pg} / \mathrm{mL}$ ) in Group 2, and

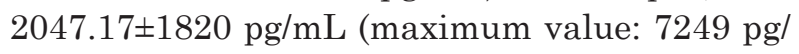
$\mathrm{mL}$, minimum value: $503 \mathrm{pg} / \mathrm{mL}$ ) in Group 3. Of the patients in the study, $80.4 \%(n=42)$ were males and $19.6 \%(n=9)$ were females. When the study group was assessed in terms of gender distribution, there were 14 males and 1 female in Group 1, 12 males and 3 females in Group 2, and 16 males and 5 females in Group 3.
The decision of surgery made by the Surgical Council of the Department of Cardiovascular Surgery with the diagnosis of coronary artery disease was determined as the study inclusion criterion. The patients with chronic obstructive pulmonary disease (COPD), chronic renal failure (CRF) and compensated CRF, acute myocardial infarction (AMI), combined valvular heart disease (VHD) and pulmonary thromboembolism, all of which cause a significant increase in NT-proBNP levels, were excluded from the study. The diagnosis of CRF that was set as one of the exclusion criteria was established if GFR was calculated below $60 \mathrm{~mL} / \mathrm{min} / 1.73 \mathrm{~m} 2$ according to CKD-epi formula (176-3).

\section{Blood Sampling:}

The blood samples were obtained upon admission at the hospital through vein puncture. The tubes with EDTA were used for automatic blood count. The blood counts were measured on a Sysmex XT1800i Hematology Analyzer (Sysmex Corporation, Kobe, Japan) Bayer ADVIA Centaur ${ }^{\text {TM }}$ immunoassay operation (Bayer Healthcare LLC, Diagnostic division, Leverkusen, Germany) was used for plasma pro-BNP level measurements (3).

\section{Surgical procedure:}

All operations were performed on cardiopulmonary bypass with the use of an intermittent global ischemia with the allowable systemic temperature between $32-34^{\circ} \mathrm{C}$. Anesthetic management and surgical techniques were the same for all patients.

Notwithstanding that exceptional circumstances have occurred, LIMA was used to graft that LAD (left anterior descending) if no complication developed, saphenous vein was used to graft diagonal branch of the LAD, saphenous vein and, if modified Allen test was negative and the patient's age was suitable, radial artery were used to graft circumflex artery $(\mathrm{Cx})$, and saphenous vein was used to graft right coronary artery (RCA).

In the postoperative period, single or multiple vasopressor agents including adrenalin, dopamine, dobutamine or noradrenalin were used if sufficient perfusion pressure could not be maintained and the mean arterial pressure remained under 80 mmHg. Dobutamine was used as the first line therapy as inotropic vasopressor agent; 
noradrenalin was used alone if arrhythmogenic effect was dobutamine could not be tolerated; and dobutamine combined with noradrenalin was used as the initial regimen if dobutamine alone has failed to produce a sufficient increase in the arterial pressure or maintain perfusion. The patients were administered adrenalin and/or dopamine if they did not respond to the use of combined noradrenalin and dobutamine. In addition, renal-dose dopamine (2-5 $\mathrm{microgram} / \mathrm{kg} / \mathrm{min})$ was administered depending on the clinical condition of the patient and as per physician's discretion in patients that were judged to have insufficient renal perfusion in the postoperative period.

Early postoperative data of the patients were collected on a daily basis in the first three days of follow-up. In the early postoperative period, blood gases analysis results, daily mean arterial blood pressure, central venous pressure, total drainage, data on the use of intra-aortic balloon pump, if available, use of inotropic agents and daily doses administered as microgram $/ \mathrm{kg} / \mathrm{min}$ were collected. The arterial blood pressure was measured using an invasive method. The amount of blood and blood products used in the first three days in the postoperative period were recorded from the intensive care unit follow-up charts.

In the postoperative period, vasopressor agents including adrenalin, dopamine, dobutamine or noradrenalin were used as a single agent or in combination, if sufficient perfusion pressure could not be maintained and the mean arterial pressure remained under $80 \mathrm{mmHg}$. Dobutamine was used as the first line therapy as inotropic vasopressor agent; noradrenalin was used alone if arrhythmogenic effect of dobutamine could not be tolerated; and dobutamine combined with noradrenalin was used as the initial regimen if dobutamine alone has failed to produce a sufficient increase in the arterial pressure or maintain perfusion. The patients were administered adrenalin and/or dopamine if they did not respond to the use of noradrenalin combined with dobutamine. In addition, renal-dose dopamine (25 microgram $/ \mathrm{kg} / \mathrm{min}$ ) was administered depending on the clinical condition of the patient and as per physician's discretion in patients that were judged to have insufficient renal perfusion in the postoperative period.

\section{Statistical Analysis}

The statistical analysis was performed using SPSS V.17 (IBM Inc.) software package. Demographic data of the patients were expressed as mean and median values. One-Way ANOVA and MannWhitney U test were used to analyze data obtained from the patients in the postoperative period. In all statistical analyzes, a $p$ value less than 0.05 was considered statistically significant.

\section{Results:}

On echocardiographic examination in the preoperative period, the mean LVEF was $56.27 \% \pm 10.39$ in the whole study group, $61.46 \% \pm 4.91$ in Group $1,60.92 \% \pm 4.57$ in Group 2, and $49.53 \% \pm 12.29$ in Group 3, and One-Way ANOVA showed statistical differences between the groups in terms of EF $(p<0.05)$ and there was a negative correlation between NT-proBNP level and EF $(p<0.05)$. The mean left ventricular enddiastolic diameter (LVEDD) was $50.20 \pm 6.96 \mathrm{~mm}$ in the whole study group, while the mean LVEDD was $46.92 \pm 4.15 \mathrm{~mm}$ in Group 1, $47.69 \pm 4.15 \mathrm{~mm}$ in Group 2, and 54.16 $\pm 7.78 \mathrm{~mm}$ in Group 3, and the differences between the groups in terms of left ventricular end-diastolic diameter were found to be statistically significant in One-Way ANOVA $(p<0.05)$. The mean left ventricular end-systolic diameter (LVESD) was $34.44 \pm 7.40 \mathrm{~mm}$ in the whole study group, while the mean LVESD was $30.77 \pm 4.62 \mathrm{~mm}$ in Group 1, 31.28 $\pm 3.36 \mathrm{~mm}$ in Group 2, and $39.05 \pm 8.55 \mathrm{~mm}$ in Group 3, and the differences between the groups in terms of left ventricular end-systolic diameter were found to be statistically significant in One-Way ANOVA $(p<0.05)$. The mean pulmonary artery pressure did not significantly differ between the study groups in One-Way ANOVA ( $p=0.09)$.

There were no significant differences between the study groups in terms of invasive arterial blood pressure, central venous pressure, output from the drain, use of inotropic agents that were collected in the first four days in the postoperative period (Table III). 
Table-I

Preoperative findings and characteristics of patients.

\begin{tabular}{|c|c|c|c|c|c|}
\hline Parameter & General & Group-1 & Group-2 & Group-3 & $P$ value \\
\hline \multicolumn{6}{|l|}{ Demographic Data } \\
\hline Age (yrs) & $63.27 \pm 8.33$ & $59.87 \pm 6.27$ & $66.07 \pm 7.85$ & $63.62 \pm 9.39$ & 0.12 \\
\hline Height $(\mathrm{cm})$ & $168.73 \pm 9.28$ & $167.87 \pm 10.66$ & $171.40 \pm 7.03$ & $167.43 \pm 9.66$ & 0.42 \\
\hline Weight (kg) & $76.84 \pm 14.20$ & $76.83 \pm 16.57$ & $81.73 \pm 12.9$ & $73.57 \pm 12.9$ & 0.24 \\
\hline \multicolumn{6}{|c|}{ Preoperative Physical Examination } \\
\hline Systolic BP & $118.92 \pm 13.9$ & $122.67 \pm 14$ & $120 \pm 16.8$ & $113.48 \pm 7.12$ & 0.3 \\
\hline Diastolic BP & $69.41 \pm 7.66$ & $69 \pm 7.12$ & $69 \pm 8.49$ & $70 \pm 7.75$ & 0.9 \\
\hline Heart Rate (minute) & $78.27 \pm 10.93$ & $76.23 \pm 11.38$ & $80.4 \pm 13.3$ & $78 \pm 9.12$ & 0.63 \\
\hline Ejection Fraction (\%) & $56.27 \pm 10.39$ & $61.46 \pm 4.91$ & $60.92 \pm 4.57$ & $49.53 \pm 12.29$ & 0.01 \\
\hline LVEDD (mm) & $50.20 \pm 6.96$ & $46.92 \pm 4.15$ & $47.69 \pm 4.15$ & $54.16 \pm 7.78$ & 0.01 \\
\hline LVESD (mm) & $34.44 \pm 7.40$ & $30.77 \pm 4.62$ & $31.28 \pm 3.36$ & $39.05 \pm 8.55$ & 0.01 \\
\hline $\mathrm{PAP}(\mathrm{mmHg})$ & $36.13 \pm 6.88$ & $24.69 \pm 5.92$ & $33.77 \pm 3.59$ & $38.74 \pm 8.43$ & 0.09 \\
\hline \multicolumn{6}{|c|}{ Preoperative Laboratory Values } \\
\hline Hemoglobin (gm/dl) & $12.91 \pm 1.35$ & $13.31 \pm 1.45$ & $13.24 \pm 0.76$ & $12.41 \pm 1.51$ & 0.08 \\
\hline Hematocrit (\%) & $39 \pm 5.16$ & $40.28 \pm 6.92$ & $40.51 \pm 3.10$ & $37.03 \pm 4.40$ & 0.07 \\
\hline Creatinine (mg/dl) & $0.86 \pm 0.19$ & $0.78 \pm 0.18$ & $0.86 \pm 0.14$ & $0.91 \pm 0.22$ & 0.14 \\
\hline GFR (ml/min) & $87.80 \pm 14.15$ & $93.93 \pm 12.62$ & $88.64 \pm 11.34$ & $82.81 \pm 15.62$ & 0.06 \\
\hline \multicolumn{6}{|l|}{ Blood gases } \\
\hline $\mathrm{pH}$ & $7.41 \pm 0.03$ & $7.41 \pm 0.03$ & $7.40 \pm 0.02$ & $7.42 \pm 0.04$ & 0.16 \\
\hline $\mathrm{PO}_{2}(\mathrm{mmHg})$ & $84.02 \pm 16.02$ & $87.73 \pm 17.66$ & $79.93 \pm 17.30$ & $84.29 \pm 13.81$ & 0.42 \\
\hline $\mathrm{PCO}_{2}(\mathrm{mmHg})$ & $36.31 \pm 4.10$ & $37.67 \pm 3.66$ & $38 \pm 3$ & $34.14 \pm 4.22$ & 0.01 \\
\hline \multicolumn{6}{|c|}{ Pumonary funtion tests } \\
\hline $\mathrm{FEV}_{1} / \mathrm{FVC}(\%)$ & $78.59 \pm 15.92$ & $84.87 \pm 11.83$ & $76.20 \pm 16.49$ & $75.81 \pm 17.42$ & 0.19 \\
\hline
\end{tabular}

BP: Blood Pressure, LVEDD: Left ventricular end-diastolic volume LVESD: Left ventricular end-systolic volume, PAP: Pulmonary Artery Pressure, GFR: Glomerular filtration rate, FVC: Force Vital Capacity

Table-II

Intraoperative variables in study population.

\begin{tabular}{lccccc}
\hline Parameter & General & Group-1 & Group-2 & Group-3 & P value \\
\hline Total CPB Time (min) & $151.70 \pm 37.41$ & $142.50 \pm 28.2$ & $154.67 \pm 37.30$ & $155.71 \pm 43.03$ & 0.58 \\
Cross-clamping time (min) & $86.67 \pm 23.01$ & $84.67 \pm 20.22$ & $88.67 \pm 22$ & $86.67 \pm 26.33$ & 0.9 \\
Number of Anastomosis & $3.27 \pm 0.8$ & $3.20 \pm 0.68$ & $3.33 \pm 0.98$ & $3.29 \pm 0.78$ & 0.9 \\
Cross-clamping Time & $28.01 \pm 10.65$ & $27.05 \pm 6.35$ & $29.58 \pm 15.03$ & $27.58 \pm 9.71$ & 0.79 \\
Per Anastomosis (min) & & & & \\
Total Length of Hospital Stay (day) & $17.08 \pm 10.80$ & $13.33 \pm 3.85$ & $16.19 \pm 15.60$ & $20.43 \pm 9.03$ & 0.14 \\
ICU Length of Stay (day) & $5.56 \pm 7.35$ & $3.53 \pm 0.64$ & $7.13 \pm 12.80$ & $5.81 \pm 3.09$ & 0.40 \\
EUROSCORE & $2.42 \pm 2.09$ & $1.33 \pm 0.98$ & $2.19 \pm 1.64$ & $3.38 \pm 2.56$ & 0.01 \\
\hline
\end{tabular}


Table-III

Use of inotropic agents (microgram/hg/min) in postoperative days.

\begin{tabular}{lccccc}
\hline Parameters & General & Group-1 & Group-2 & Group-3 & P value \\
\hline IT (hour) & $10.6 \pm 8.1$ & $8.12 \pm 7.28$ & $9.82 \pm 8.35$ & $12.94 \pm 8.20$ & 0.19 \\
Postoperative Day 0 & & & & & \\
Adrenalin & $0.1 \pm 0.7$ & None & None & $0.24 \pm 1.09$ & 0.5 \\
Dopamine & $0.08 \pm 0.56$ & None & $0.27 \pm 1.03$ & None & 0.31 \\
Dobutamine & $2.1 \pm 3.1$ & $1.73 \pm 2.71$ & $1.73 \pm 2.81$ & $2.62 \pm 3.38$ & 0.59 \\
Noradrenalin & $1.35 \pm 3.45$ & $0.67 \pm 1.50$ & $0.67 \pm 1.50$ & $2.33 \pm 4.67$ & 0.24 \\
Postoperative Day 1 & & & & \\
Adrenalin & $0.08 \pm 0.57$ & None & None & $0.19 \pm 0.85$ & 0.51 \\
Dopamine & $0.08 \pm 0.56$ & None & $0.27 \pm 1.13$ & None & 0.31 \\
Dobutamine & $2.10 \pm 3.01$ & $1.73 \pm 2.71$ & $1.73 \pm 2.81$ & $2.62 \pm 3.38$ & 0.59 \\
Noradrenalin & $1.35 \pm 3.45$ & $0.67 \pm 1.50$ & $0.67 \pm 2.58$ & $2.33 \pm 4.67$ & 0.24 \\
Postoperative Day 2 & & & & & \\
Adrenalin & $0.09 \pm 0.58$ & None & None & $0.21 \pm 0.92$ & 0.49 \\
Dopamine & None & None & None & None & - \\
Dobutamine & $1.5 \pm 3.35$ & $0.53 \pm 2.07$ & $0.92 \pm 2.15$ & $2.52 \pm 4.33$ & 0.17 \\
Noradrenalin & $0.62 \pm 2.21$ & None & $0.13 \pm 0.52$ & $1.47 \pm 3.36$ & 0.09 \\
\hline
\end{tabular}

IT - Intubation time

\section{IT: intubation time,}

At postoperative day 0 , the mean adrenalin, dopamine, dobutamin, and noradrenalin consumptions were $0.1 \pm 0.7 \mathrm{microgram} / \mathrm{kg} / \mathrm{min}$, $0.08 \pm 0.56 \mathrm{microgram} / \mathrm{kg} / \mathrm{min}, \quad 2.1 \pm 3.01$ $\mathrm{microgram} / \mathrm{kilogram} / \mathrm{min}$, and $1.35 \pm 3.45$ microgram $/ \mathrm{kg} / \mathrm{min}$, respectively. There were no statistically significant differences between the groups in terms of the use of adrenalin $(p=0.50$, $p>0.05)$, dopamine ( $p=0.31 p>0.05)$, dobutamine $(p=0.59 p>0.05)$, and noradrenalin $(p=0.24 p>0.05)$ at postoperative day 0 .

The doses of inotropic agents used at postoperative days 1 and 2 did not show significant differences between the groups for the three inotropic agents.

The mean EUROSCORE risk score was $2.42 \pm 2.09$ in the study group, and there was a positive correlation between EUROSCORE risk score and serum NT-proBNP levels $(\mathrm{p}=0.01 \mathrm{p}<0.05)$.

\section{Discussion:}

In this study, we found that there were no significant differences between the study patients divided into three groups depending on NT-proBNP levels as low NT-proBNP $(<100 \mathrm{pg} / \mathrm{mL})$ group, high NT-proBNP (>500 pg/mL) group, and moderately high NT-proBNP group (NT-proBNP $<500$ pg/mL and $>100 \mathrm{pg} / \mathrm{mL}$ ) in terms of use of inotropic agents in the first four days in the postoperative period. We also found no significant difference in terms of invasive arterial blood pressure, central venous pressure, output from the drain according to NTproBNP levels.

The monitoring of plasma BNP and NT-proBNP levels provide important information about hemodynamic status during follow-up in the emergency setting particularly in patients exhibiting cardiac arrhythmia and those who previously underwent cardiac transplantation. These parameters can be used in intraoperative risk assessment during surgeries such as coronary artery bypass grafting and studies have used these parameters to determine prognosis in the postoperative period. ${ }^{4}$ In one of these studies published by Eliasdottir et al. ${ }^{5}$ patients with a prolonged stay in the intensive care unit and patients who died in the first 28 days after the operation had significantly higher NT-proBNP levels in the preoperative period, and in addition, these patients also displayed significantly higher need for the use of intravenous inotropic agents, higher need for IABP support and higher risk of developing renal insufficiency. These publications suggest that preoperative plasma NT-proBNP levels might be of benefit in predicting the course of stay in the intensive care unit, length of stay in 
the intensive care unit and total length of hospital stay. ${ }^{6}$ In the study by Eliasdottir et al., there was also a strong correlation between preoperative NTproBNP levels and EUROSCORE risk assessment scores. ${ }^{5}$ Similar to this study, there was a positive correlation between EUROSCORE risk score and serum NT-proBNP levels in our study. Also, Hutfless et al. showed a significant relationship between preoperative and peak postoperative BNP levels and prolonged hospital stay and mortality rate at one year. ${ }^{6}$

NT-proBNP levels relate to heart failure. Also, inotropic agents are used in the treatment of heart failure. Therefore, elevated NT-proBNP levels are expected in patients receiving inotropic agents. In the study by Morimoto et al., there was a significant relationship between inotropic agents and NTproBNP levels. ${ }^{7}$ The patients with elevated NTproBNP levels were supported with inotropic agents. The rate of inotropic agent use was also higher. Hutfless et al. evaluated the use of intraaortic balloon pump and NT-proBNP levels in patients undergoing cardiac surgery, and patients with elevated NT-proBNP levels in the preoperative period had significantly increased rate of intra-aortic balloon pump. ${ }^{6}$ Intra-aortic balloon pump was used only in 3 patients all of whom were in Group 3. Schachner et al. evaluated the relationship between prolonged mechanical ventilation time and preoperative serum NTproBNP levels, and they found that patients with NT-proBNP levels above $502 \mathrm{pg} / \mathrm{ml}$ in the preoperative period displayed significantly higher need for mechanical ventilator support, longer stay in the intensive care unit, IABP, and higher hemofiltration support and higher rates of $\mathrm{AF}$; however, the present study did not show significant differences between the groups in terms of mechanical ventilator support. ${ }^{8}$

In another study, Chello et al. evaluated plasma ANP and BNP levels in patients with low left ventricular ejection fraction, and although BNP was showed to be an important marker in the recovery period after open heart surgery, their study was not powered to draw more accurate conclusions due to lack of a control group with preserved left ventricular function. ${ }^{9}$ In another study, Provenchere et al. showed that serum BNP levels were important in predicting the outcomes at one year in patients undergoing cardiac surgery; however, they also showed that elevated plasma BNP levels were associated with a 12-fold increase in the risk of developing postoperative heart failure and only LVEF showed a significant correlation with 1-year survival in statistical analysis. ${ }^{10}$

Some limitations of the study need to be addressed: first of all, the number of patients is relatively small. Another limitation of this study is the different gender characteristics between patients groups.

The results of the present study are inconsistent with the data in the literature, and this was attributed to the functional revival of the contractile myocardial tissue after revascularization and maintenance of arterial blood pressure at sufficient level to avoid the use of inotropic agents. No further assessments could be made to support this argument due to the fact that myocardial viability was not evaluated in the preoperative period. We believe that larger studies are needed to confirm these findings.

\section{Conflict of Interest - None.}

\section{References:}

1. Munagala VK, Burnett JC Jr, Redfield MM. The natriuretic peptides in cardiovascular medicine. Curr Probl Cardiol 2004; 29: 707-769.

2. Saribulbul O, Alat I, Coskun S, Apaydin AZ, Yagdý T, Kýlýccýoglu M, et al. The role of brain natriuretic peptide. Tex Heart Inst $J$ 2003; 30: 298-304.

3. Park JH, Shin GJ, Ryu JI, Pyun WB. Postoperative Btype natriuretic Peptide levels associated with prolonged hospitalization in hypertensive patients after noncardiac surgery. Korean Circ J 2012; 42: 521-527.

4. Ciccone MM, Cortese F, Gesualdo M, Riccardi R, Di Nunzio D, Moncelli M, et al. A novel cardiac bio-marker: ST2: a review. Molecules 2013; 18: 15314-15328.

5. Eliasdottir SB, Klemenzson G, Torfason B, Valsson F. Brain natriuretic peptide is a good predictor for outcome in cardiac surgery. Acta Anaesthesiol Scand 2008; 52: $182 \mathrm{e} 7$.

6. Hutfless R, Kazanegra R, Madani M, Bhalla MA, TuluaTata A, Chen A, et al. Utility of b-type natriuretic peptide in predicting postoperative complications and outcomes in patients undergoing heart surgery. $\mathrm{J} \mathrm{Am} \mathrm{Coll}$ Cardiol 2004; 43: 1873e9.

7. Morimoto K, Mori T, Ishiguro S. Perioperative changes in plasma brain natriuretic peptide concentrations in patients undergoing cardiac surgery. Surg Today 1998; 28: $23-29$. 
8. Schachner T, Wiedemann D, Fetz H, Laufer G, Kocher A, Bonaros N. Influence of preoperative serum Nterminal pro-brain type natriuretic peptide on the postoperative outcome and survival rates of coronary artery bypass patients. Clinics (Sao Paulo) 2010; 65: 1239-1245.

9. Chello M, Mastroroberto P, Perticone F, Cirillo F, Bevacqua E, Olivito S, et al. Plasma levels of atrial and brain natriuretic peptides as indicators of recovery of left ventricular systolic function after coronary artery bypass. Eur J Cardiothorac Surg 2001; 20: 140-146.

10. Provenchere S, Berroeta C, Reynaud C, Baron G, Poirier I, Desmonts JM, et al. Plasma brain natriuretic peptide and cardiac troponin I concentrations after adult cardiac surgery: association with postoperative cardiac dysfunction and 1-year mortality. Crit Care Med 2006; 34: $995-1000$. 